\title{
La Recreación en los adolescentes. Su importancia en el desarrollo biopsicosocial: Una aproximación teórica-descriptiva.
}

\author{
Recreation in adolescents. Its importance in biopsychosocial development: \\ A theoretical-descriptive approach.
}

Cristhian Steven Morillo Hurtado. ${ }^{1}$, Stevens Mauricio Cajiao Narváez. ${ }^{2}$ \& María Lorena Sandoval Jaramillo. ${ }^{3}$

Introduction. The biopsychosocial development and comprehensive care of adolescents includes multiple actions, within which Recreation occupies a fundamental place; Especially if we bear in mind that worldwide the indices of chronic non-communicable diseases are growing disproportionately, many of these due to the scarce practice of physical, sports and recreational activities. Objective. Systematize the theories related to the importance of Recreation in the biopsychosocial development of adolescents for the improvement of intervention strategies and implementation in practice. Methodology. The research followed a descriptive methodology, supported by the Systematic Literature Review (RSL), with the use of theoretical methods and the documentary review of different sources and databases between 2000 and 2021. Results. 1. It was possible to delve into the generalities and importance of Recreation and its incidence in comprehensive care and biopsychosocial development of adolescents, establishing general guidelines to consider for the implementation in practice of intervention strategies through Recreation, as an essential component for the progress of physical, motor, social, behavioral and cognitive skills of adolescents. 2. The need to work on the selection of recreational activities from the perspective of diagnosing the tastes, motivations and interests of adolescents was confirmed. Conclusions. Adolescence is a time of great change that requires permanent attention. The inclusion and participation in recreational activities properly planned and in correspondence with the tastes and preferences of adolescents constitute an effective alternative to prevent the appearance of possible

\footnotetext{
${ }^{1}$ Universidad de las Fuerzas Armadas. (ESPE), Carrera: Pedagogía de la Actividad Física y Deporte csmorillo@espe.edu.ec ORCID ID: 0000-0002-7166-6323

${ }^{2}$ Universidad de las Fuerzas Armadas (ESPE) smcajiao@espe.edu.ec ORCID ID: 0000-0002-6017-215X

${ }^{3}$ Universidad de las Fuerzas Armadas. (ESPE), Carrera: Pedagogía de la Actividad Física y Deporte mlsandoval1@espe.edu.ec ORCID ID: 0000-0003-1532-1862
} 
diseases and enhance their biopsychosocial development. The joint responsibility that is achieved by all those involved in developing quality recreational strategies, including adolescents, will guarantee the success and fulfillment of the objectives to be achieved.

Keywords: recreation, adolescents, biopsychosocial development, comprehensive care, recreational strategies.

\section{Resumen.}

Introducción. El desarrollo biopsicosocial y la atención integral de los adolescentes incluye múltiples acciones, dentro de las cuales la Recreación ocupa un lugar fundamental; máxime si tenemos presente que a nivel mundial crecen desproporcionalmente los índices de enfermedades crónicas no trasmisibles, muchas de estas por la escasa práctica de actividades físicas, deportivas y recreativas. Objetivo. Sistematizar las teorías relacionadas con la importancia de la Recreación en el desarrollo biopsicosocial de los adolescentes para el perfeccionamiento de las estrategias de intervención e implementación en la práctica. Metodología. La investigación siguió una metodología descriptiva, sustentada en la Revisión Sistemática de Literatura, (RSL), con el empleo de métodos teóricos y la revisión documental de diferentes fuentes y bases de datos comprendidas entre el 2000 y el 2021. Resultados. 1. Se logró profundizar en las generalidades e importancia de la Recreación y su incidencia en la atención integral y el desarrollo biopsicosocial de los adolescentes, llegando a establecer orientaciones generales a considerar para la implementación en la práctica de las estrategias de intervención mediante la Recreación, como componente esencial para el progreso de habilidades físico motoras, sociales, conductuales y cognitivas de los adolescentes. 2. Quedó confirmado la necesidad de trabajar la selección de actividades recreativas desde la perspectiva del diagnóstico en cuanto a gustos, motivaciones e intereses de los adolescentes. Conclusiones. La adolescencia es una etapa de grandes cambios que requiere de atención permanente. La inclusión y participación en actividades recreativas adecuadamente planificadas y en correspondencia con los gustos y preferencias de los adolescentes constituyen una alternativa eficaz para para prevenir la aparición de posibles enfermedades y potenciar su desarrollo biopsicosocial. La corresponsabilidad que se logre por parte de todos los implicados en desarrollar estrategias recreativas de calidad, incluyendo los adolescentes, garantizará el éxito y cumplimiento de los objetivos a lograr.

Palabras clave: recreación, adolescentes, desarrollo biopsicosocial, atención integral, estrategias recreativas.

\section{Introducción}

La urgencia por recuperar lo perdido en cuanto a hábitos saludables y mayor calidad de vida sigue constituyendo un aspecto de exclusiva atención para todos los entes gubernamentales, de salud, educativos y deportivos. Los avances científicos- 
tecnológicos han posibilitado la mejora de muchos aspectos de la vida moderna, pero también han dado lugar al crecimiento de una sociedad cada vez más sedentaria, con altos índices de obesidad y enfermedades crónicas no trasmisibles; muchas de estas ocasionadas por los escasos hábitos para la práctica de actividades físicas-deportivas y recreativas desde edades tempranas, trayendo consigo grandes afectaciones en la salud y en el desarrollo integral en general.

En contraposición con estas tendencias, ha quedado demostrado que la práctica sistemática de actividades recreativas constituye una alternativa efectiva para una mejor calidad de vida en las diferentes edades. Es por ello que convertir la Recreación en un componente básico de nuestra vida sigue siendo una acción prioritaria a consolidar en las diferentes edades.

En esta línea de pensamientos se coincide con Sigler, et al.,(2020), al afirmar que: "La recreación como elemento de la cultura del hombre debe ser tratada con un nivel significativo en el proceso de formación de la personalidad. Apuntan los autores mencionados que la recreación física y sus disímiles actividades tienen una gran repercusión en la vida de las personas, por lo que en su desarrollo necesitan de una adecuada organización y control”. (Pág 1)

En el caso de los adolescentes la realización de actividades recreativas es fundamental para potenciar su desarrollo biopsicosocial de forma integral. Nótese que en el informe emitido por la Organización Mundial de la Salud (OMS) en marzo del 2021, en conmemoración al Día Mundial de la Obesidad se expone que la obesidad ha alcanzado proporciones epidémicas a nivel mundial. Estimándose que desde 1975 esta enfermedad se ha triplicado. Se señala en el informe, que en el 2016 se incrementó a 1900 millones de adultos y más de 340 millones de niños y adolescentes entre 5 a 19 años presentaban indicadores de sobrepeso u obesidad, aumentando en un 18\% en comparación con 1975. Destacan que este crecimiento ha sido similar en ambos sexos con un $18 \%$ en las niñas y un $19 \%$ en los niños.

El análisis de estos indicadores en Ecuador también resulta alarmante. Llama la atención los datos emitidos por la UNICEF, al indicar que tanto en los niños como en los adolescentes los índices de sobrepeso y obesidad han ido en aumento, destacan que: 1 de cada 3 niños en edad escolar y 1 de cada 4 adolescentes ya registra sobrepeso. Los estudios de Ramos et al.,(2015), aseveran la fuerte preocupación existente en Ecuador por el acentuación de niños y adolescentes con presencia de sobrepso, obesidad y otras enfermedades, todas prevenibles si se practicaran actividades físicas-recreativas y se fomentaran hábitos adecuados de alimentación.

Según Ladinez, (2020), en el caso de Ecuador desde el Estado nacional se sostiene un modelo vinculado al deporte y la recreación que entiende al deporte, la actividad física y la recreación como derechos que deben ser garantizados en todo el territorio nacional en busca no solo de la integración e inclusión sino también del desarrollo humano integral. En el contexto ecuatoriano desde la propia Constitución de la República se norma todo lo 
relacionado con el derecho a la Recreación, el Tiempo Libre en función de una mejor calidad de vida en los distintos grupos etarios sin distinción de sexo, edad o condición social.

En correspondencia con el marco legal existente se asume la importancia y reconocimiento que se da desde la propia Constitución de la República a la práctica sistemática de las actividades físicas con especial interés en el uso adecuado del tiempo Libre y la realización de actividades recreativas que sean placenteras y beneficiosas para la salud

Ahora bien, independientemente de los variados esfuerzos desarrollados por los entes gubernamentales, políticos, educativos y sociales, así como de las investigaciones realizadas a nivel internacional relacionadas con los beneficios de la Recreación para el desarrollo biopsicosocial de los adolescentes, aun resultan insuficientes en el contexto nacional los estudios que desde un posicionamiento teórico profundo permitan sistematizar la importancia de la Recreación como componente esencial en el desarrollo biopsicosocial de los adolescentes; todo lo cual incide negativamente en la implementación de acciones oportunas para el desarrollo de estrategias de intervención y programas recreativos en estas edades.

Lo anterior crea una brecha epistémica que se concreta en la necesidad de un abordaje teórico más amplio para poder elaborar e implementar estrategias de intervención en la práctica que sean oportunas, equitativas y diversas y que a su vez contribuyan al desarrollo biopsicosocial y la atención integral de los adolescentes en los diferentes contextos, desde la perspectiva de la Recreación.

Todo lo señalado justifica la necesidad de sistematizar las teorías precedentes en el ámbito ecuatoriano en relación con la importancia de la Recreación en el desarrollo biopsicosocial de los adolescentes, es por ello que cabe preguntarnos ¿Cómo incide la Recreación en el desarrollo biopsicosocial de los adolescentes? De ahí que el Objetivo General de la presente investigación se concrete en: Sistematizar las teorías relacionadas con la importancia de la Recreación en el desarrollo biopsicosocial de los adolescentes para el perfeccionamiento de las estrategias de intervención e implementación en la práctica.

\section{Un acercamiento a las generalidades de la Recreación. Conceptualización e importancia.}

El Diccionario de la Lengua Española en su versión del (2020), indica que el término Recreación, proviene del lat. Recreatio, onnis - relacionando este con la acción y efecto de recrear. Diversión para el alivio del trabajo.

En este orden de ideas autores como Pérez, (1997) \& Aguilar, (2004), señalan que la Recreación está conformada por el conjunto de fenómenos y relaciones que se dan como resultado del tiempo libre. Explican que se desarrolla mediante la realización de diferentes actividades físicas, deportivas cognoscitivas e incluso actividades culturales y artísticas. 
Enfatizan además en que la Recreación provoca felicidad, satisfacción y algo a nuestro modo fundamental es que, la Recreación contribuye al desarrollo integral de la personalidad, motivando el crecimiento físico y espiritual del individuo.

Según, Ramos et al., (2011), la recreación se define como la acción y efecto de recrear. Señalan que es necesario verla como una necesidad y una oportunidad de los seres humanos. Al relacionarla con otros términos mencionan a todos aquellos que tienen que ver con divertirse, alegrarse, deleitarse, entretenerse. Apuntan que se trata de un proceso que busca la distracción en medio del trabajo y de las obligaciones cotidianas, tanto en lo espiritual, lo físico, técnico, cultural o profesional. A nuestro criterio lo más importante está en lo concerniente a lo que aporta la recreación desde el punto de vista del desarrollo personal, comunitario y social.

Por su parte Waichman (2015), plantea que el elemento que define y caracterizan la recreación está dado por aquellas actividades que provocan placer, diversión. Comparte con otros autores al explicar que se trata de aquellas actividades que permiten el reencuentro consigo mismo y favorecen el desarrollo integral, la integración a la sociedad, la expresión y creatividad; por supuesto acorde a las condiciones y antecedentes étnicos, socioculturales y el grupo atareo al cual pertenece.

En esta misma línea es conveniente explicar que desde épocas primitivas el hombre ha venido realizando diferentes actividades recreativas que le han permitido a partir de las perspectivas individuales y sociales de cada etapa poder expandir sus potencialidades hacia nuevas formas de desarrollo y participación. Concordamos con Herrera, (2016), en que los individuos que practican actividades físicas-recreativas, independiente a cuáles sean, poseen una mejor predisposición, y están acompañados de un mayor bienestar psicológico.

Lo cierto es que la participación en actividades recreativas constituye una necesidad prioritaria para todos los seres humanos sin distinción de condición social, étnica, género o incluso capacidad.

Razo, (2017), subraya que a nivel mundial las actividades recreativas han ocupado un lugar excepcional para contribuir con el progreso de habilidades físico motoras, sociales y cognitivas del adolescente, el aspecto recreativo ha venido creciendo a medida que se presenta en los pueblos como un factor primordial en el desarrollo social e individual. (Razo, 2017, pág. 2)

Múltiples estudios a nivel internacional permiten sustentar la importancia de la Recreación en las diferentes edades. En este sentido Lezama, (2000), seguido por Mateo (2014), confirman en sus investigaciones la importancia y beneficios que ofrece la práctica sistemática de actividades recreativas, tanto desde el punto de vista físico como también psicológico y social. En correspondencia con ello Mateo, (2014), explica que las actividades recreativas ocupan un lugar fundamental, influyendo en el desarrollo personal y también en el desarrollo educativo de los practicantes. Se coincide con el autor de referencia en que las actividades recreativas tanto desde el ámbito personal como 
educativo se complementan y permiten la utilización adecuada del tiempo libre, favoreciendo la adquisición de conocimientos y la formación de valores.

Si resulta importante considerar lo explicado por el autor precedente, al señalar que siempre es necesario antes de desarrollar cualquier tipo de actividades recreativas conocer las características, tipología y beneficios de las actividades recreativas, así como la edad e interés de los posibles participantes. Esto con el objetivo de garantizar la calidad y beneficios de la recreación.

De igual manera los aportes de Tocto et al., (2015) enfatizan en la importancia de la Recreación en los estudiantes universitarios, destacando que es necesario, motivarlos hacia una práctica sistemática de actividades físico-deportivas y recreativas, previa valoración de sus gustos y preferencias en correspondencia con el respeto a su tiempo libre.

Por su parte Calero et al., (2016), (a), en su estudio sobre las actividades recreativas y su influencia en la autoestima en el adulto mayor concluyen que las actividades físicorecreativas son determinantes para el mantenimiento de la salud física y mental, argumentando que son un medio idóneo para mejorar la calidad de vida e incrementar la autoestima de los adultos mayores.

Como puede apreciarse en las investigaciones realizadas tanto a nivel internacional como nacional ha quedado fundamentada la valía de la recreación como componente esencial en las diferentes edades y épocas sociales.

De lo anterior se derivan dos ideas muy valiosas para nuestro estudio, la primera nos indica que las actividades recreativas ocupan un lugar importante para el bienestar físico, psicológico y social, de todos los seres humanos, convirtiéndose en una necesidad a suplir, independientemente a su edad o condición. La segunda idea es precisamente la planteada por Waichman (2015), al señalar que la Recreación es vista en dos variantes: La recreación como compensación del trabajo (o las obligaciones externas) y la recreación como actividad o conjunto de actividades

\section{Relación de la Recreación con el desarrollo biopsicosocial de los adolescentes.}

Se comparte con Borras, et al., (2017), en que la adolescencia es una etapa de grandes cambios biológicos, psicológicos y sociales. En esta etapa de la vida además de las transformaciones que se suceden propias de la edad, el adolescente recibe la influencia del mundo que le rodea favoreciendo el desarrollo de nuevas oportunidades. De ahí que se coincide con los autores precedentes en la importancia que tiene esta etapa y en la necesidad de continuar desarrollando investigaciones que permitan profundizar en las características de los adolescentes con la intención de prevenir la aparición de enfermedades o lo que es peor conductas desajustadas.

Dado los cambios y características de la adolescencia esta es considerada una etapa de la vida de los seres humanos que requiere de una atención especial por parte de la familia e 
instituciones educativas y sociales, es por esto extremadamente importante comprender la incidencia de la Recreación en estas edades. Diversos estudios muestran su utilidad en los procesos formativos y de desarrollo de la personalidad. Lo explicado nos articula con las teorías de Dos Reis, (2016), al señalar que los adolescentes se encuentran en la clasificación de grupos de alta vulnerabilidad, estos precisan ser atendidos con marcado interés, ello implica promover el desarrollo de investigaciones y debates conducentes a la realización de mejores programas y estrategias de intervención en estas edades.

Sin embargo, uno de los principales problemas que actualmente se suscita en el contexto de la Recreación en los adolescentes está dado por los limitados estudios que profundicen en la importancia de la Recreación en esta etapa y que a su vez permitan valorar los procesos de diagnóstico para identificar los interés, gustos y motivaciones que presentan los adolescentes en cuanto a actividades recreativas, generándose un distanciamiento entre lo que se oferta y los gustos y preferencias de los propios adolescentes.

Esto como es lógico incide negativamente en el desarrollo biopsicosocial de los adolescentes, ya que en muchas ocasiones la participación en las actividades recreativas es restringida y en muchas ocasiones se realizan sin tener en cuenta sus intereses.

En esta misma línea, el estudio realizado por Chávez \& Sandoval (2014), es muy significativo; los autores insisten en que es muy necesario determinar las características de la población a intervenir, señalando que no se trata de proponer proyectos de Actividad Física o de uso adecuado del Tiempo Libre, según intereses políticos o gubernamentales. Manifiestan que se requiere determinar previamente instrumentos de evaluación validados que permitan tomar las decisiones específicas para el sector a intervenir y plantear propuestas o acciones de trabajo que respondan a las necesidades $\mathrm{y}$ características de los mismos.

Lo anterior adquiere gran valor, justamente porque para lograr el perfeccionamiento de las estrategias de intervención mediante los diferentes programas y proyectos recreativos, es necesario contemplar los gustos, intereses, motivaciones, juicios y valoraciones de los posibles participantes. Todo ello será posible si se trabaja sobre la base del diagnóstico.

Siguiendo estas ideas Sosa (2015), llama la atención sobre las características de los modelos recreativos, explicando que en su mayoría tienen una tendencia a la pasividad y al consumismo, especialmente por no considerar el papel protagónico del hombre como ser social en el manejo adecuado del tiempo libre.

En este orden es oportuno evaluar que la actualidad ecuatoriana evidencia la necesidad de fomentar la práctica de actividades recreativas sobre todo en la etapa de la adolescencia, dado los propios cambios que se dan en estos rangos de edades. Hernández $\&$ Kelly (2014), confirman que son escasas las experiencias que se tienen en cuanto a la participación de los estudiantes que asisten a las diferentes unidades educativas que muestren vivencias de experiencias recreativas placenteras. Destaca también en la consulta realizada los aportes de Castillo (2016) y Torres, et al (2016) al señalar que las actividades recreativas son una alternativa viable y eficiente para mejorar el 
comportamiento social de los adolescentes estudiados.

Somos del criterio que la efectividad y solución a la situación que presenta hoy la Recreación para los adolescentes está precisamente en lograr un equilibrio entre lo que ellos desean y necesitan y lo que se ofrece mediante los diferentes programas y estrategias de intervención.

\section{Metodología}

El desarrollo de la investigación siguió una metodología de tipo descriptiva, basada en la revisión de la literatura comprendida entre los años 2000 y 2020; misma que se encuentra ubicada en diferentes bases de datos como: Google Académico, Latindex, Dialnet, Scopus, así como Tesis realizadas en universidades nacionales e internacionales. Los métodos teóricos que facilitaron el desarrollo investigativo fueron: el histórico-lógico, el analítico - sintético, inductivo - deductivo y la revisión documental. Para organizar la búsqueda bibliográfica fue necesario primero planificar que se realizaría, seguidamente analizar los registros y bases de datos más importantes y que más relación tuvieran con el tema de investigación, para finalmente establecer los resultados; los cuales se basaron en los estudios más significativos y de mayor importancia para nuestra investigación. Para lograr lo anterior se determinó como palabras claves: Recreación, Adolescentes y Desarrollo Biopsicosocial, resultando. la Recreación y la Adolescencia ( $\mathrm{R}-\mathrm{A}$ ), los conectores que condujeron todo el proceso investigativo bajo los criterios de inclusión establecidos tales como:

1. Estudios e investigaciones comprendidas en el rango de tiempo entre el 2000 y el 2020, relacionados con la Recreación y su importancia en las diferentes etapas de la vida.

2. Revisiones teóricas o investigaciones experimentales en idioma español o inglés relacionadas con el tema de investigación.

3. Aportes y sistematizaciones encontradas en tesis, artículos científicos, libros vinculados con el tema de la Recreación en la Adolescencia.

Los criterios de exclusión básicamente estuvieron relacionados con la exclusión de investigaciones o aportes realizados en un rango de tiempo diferente al previsto y que dado sus aportes no tuvieran significación para el trabajo realizado. Todo ello permitió dar seguimiento a la calidad y rigor científico del trabajo que se presenta.

\section{Resultados}

La Revisión Sistemática de la Literatura (RSL) y de fuentes precedentes permitió tener un acercamiento teórico descriptivo en relación con el tema. La misma contempló un total de 26 fuentes de las cuales 8 fueron altamente potenciales, siendo estos los estudios que mayor significación y relación tuvieron con el tema investigado, cumpliéndose de este modo con los criterios de inclusión y exclusión 
establecidos por el investigador para la realización del trabajo.

Obsérvese en la Tabla No. 1, los principales aportes encontrados en los estudios referenciados y que se relacionan con el tema investigado.

Tabla No 1. Resultados de la búsqueda realizada Artículos referentes para la investigación entre los años (2000- 2021).

\begin{tabular}{|c|c|c|c|c|}
\hline Nombre del autor & Titulo & Año & $\begin{array}{l}\text { Base de publicación y } \\
\text { tema }\end{array}$ & Aportes \\
\hline $\begin{array}{l}\text { Chávez, E., \& Sandoval, } \\
\text { L. }\end{array}$ & $\begin{array}{l}\text { Estudio del tiempo } \\
\text { libre de los } \\
\text { adolescentes } \\
\text { ecuatorianos del } \\
\text { Colegio Nacional } \\
\text { Juan de Salinas de } \\
\text { Sangolquí }\end{array}$ & (2014) & $\begin{array}{l}\text { Revista Digital. } \\
\text { EFDeportes.com, / } \\
\text { Buenos Aires, Año18, No } \\
\text { 188, enero de 2014. } \\
\text { http://www.efedeportes.c } \\
\text { om }\end{array}$ & $\begin{array}{l}\text { El estudio resultó muy } \\
\text { importante para la } \\
\text { investigación, el mismo } \\
\text { tuvo como objetivo } \\
\text { evaluar el uso del tiempo } \\
\text { libre activo y pasivo de } \\
\text { un grupo de estudiantes } \\
\text { y su repercusión en el } \\
\text { nivel de sedentarismo. } \\
\text { Para ello los autores } \\
\text { adaptaron una encuesta } \\
\text { aplicada en otros países } \\
\text { y el PAQ-A (Physical } \\
\text { Activity Questionnaire } \\
\text { for Adolescents). Los } \\
\text { resultados evidencian } \\
\text { que el mayor tiempo es } \\
\text { utilizado en actividades } \\
\text { pasivas, mientras que } \\
\text { solo el 19,91\% lo } \\
\text { dedican a actividades } \\
\text { dinámicas lo que } \\
\text { demuestra un mal uso } \\
\text { del tiempo libre } \\
\text { repercutiendo en los } \\
\text { indicadores de } \\
\text { sedentarismo. } \\
\text { Concluyen con la } \\
\text { propuesta de un sistema } \\
\text { de Actividades. }\end{array}$ \\
\hline Mateo, JL. & $\begin{array}{l}\text { Las actividades } \\
\text { recreativas: sus } \\
\text { características, } \\
\text { clasificación y } \\
\text { beneficios }\end{array}$ & 2014) & $\begin{array}{l}\text { EFDeportes.com. Revista } \\
\text { Digital. Buenos aires, año } \\
\text { 19, No 196, septiembre de } \\
\text { 2014. http:// } \\
\text { www.efdeportes.com }\end{array}$ & $\begin{array}{l}\text { El estudio desarrollado } \\
\text { define, clasifica y } \\
\text { determina las } \\
\text { actividades recreativas, } \\
\text { según áreas, enfatizando } \\
\text { en los múltiples } \\
\text { beneficios que tienen las } \\
\text { actividades recreativas } \\
\text { en las diferentes edades. }\end{array}$ \\
\hline $\begin{array}{l}\text { Ramos A E., Ojeda, R., } \\
\text { Báez, D., Nuñez, E., }\end{array}$ & $\begin{array}{l}\text { La Recreación: } \\
\text { necesidad y } \\
\text { oportunidad para } \\
\text { el desarrollo } \\
\text { humano desde y } \\
\text { para las } \\
\text { comunidades }\end{array}$ & 2011 & $\begin{array}{l}\text { EFDeportes.com, Revista } \\
\text { Digital. Buenos Aires, } \\
\text { Año 16, No } 160 \text {, } \\
\text { septiembre de } 2011 . \\
\text { http:// } \\
\text { www.efedeportes.com }\end{array}$ & $\begin{array}{lcr}\text { La } & \text { investigación } \\
\text { reflexiona } & \text { sobre } & \text { la } \\
\text { importancia } & \text { de } & \text { la } \\
\text { Recreación } & \text { y } & \text { sus } \\
\text { beneficios } & \text { en } & \text { el } \\
\text { desarrollo } & \text { humano, } \\
\text { desde la base del } \\
\text { desarrollo comunitario y } \\
\text { del movimiento. }\end{array}$ \\
\hline $\begin{array}{l}\text { Calero, S., Fernández, A., } \\
\text { \& Castillo de la Rosa, FL., } \\
\text { 9-14. (b) }\end{array}$ & $\begin{array}{l}\text { Recreation } \\
\text { activities to } \\
\text { improve social } \\
\text { behavior. Study in } \\
\text { children and } \\
\text { adolescents aged }\end{array}$ & (2016) & $\begin{array}{l}\text { Revista Cubana de } \\
\text { Medicina General } \\
\text { Integral,32(3) } \\
\text { Recuperado en } 05 \text { de } \\
\text { mayo de 2021, de } \\
\text { http://scielo.sld.cu/scielo. } \\
\text { php?script=sci_arttext\&pi }\end{array}$ & $\begin{array}{l}\text { La investigación resultó } \\
\text { altamente importante } \\
\text { para el estudio que se } \\
\text { presenta. La misma } \\
\text { evalúa el efecto de } 15 \\
\text { actividades recreativas } \\
\text { en el comportamiento }\end{array}$ \\
\hline
\end{tabular}




\begin{tabular}{|c|c|c|c|c|}
\hline & & & $\begin{array}{l}\frac{\mathrm{d}=\mathrm{S} 0864}{21252016000300008 \& \ln } \\
\mathrm{g}=\mathrm{es} \& \mathrm{tlng}=\mathrm{en} . \\
\text { Indexada LILACS, } \\
\text { SCOPUS, SciELO y } \\
\text { Latindex }\end{array}$ & 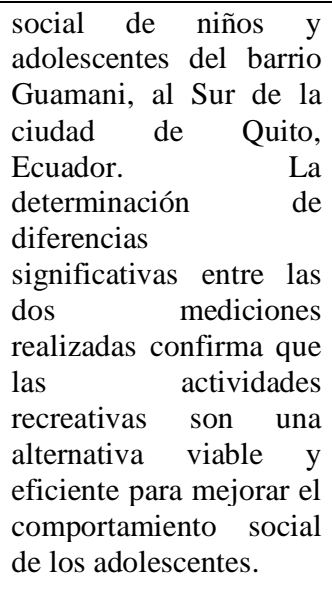 \\
\hline $\begin{array}{l}\text { Ramos, P., Carpio, } \\
\text { A., Delgado, V., } \\
\text { Villavicencio, V., }\end{array}$ & $\begin{array}{lr}\text { Sobrepeso } & \text { y } \\
\text { obesidad } & \text { en } \\
\text { escolares } & y \\
\text { adolescentes } & \text { del } \\
\text { área urbana de la } \\
\text { ciudad } & \text { de } \\
\text { Riobamba, } & \\
\text { Ecuador. } & \end{array}$ & $\begin{array}{l}(2015) \\
\cdot\end{array}$ & $\begin{array}{l}\text { Revista Española de } \\
\text { Nutrición Humana y } \\
\text { Dietética, 19(1), 21- } \\
\text { 27. https://dx.doi.org/10.1 } \\
\text { 4306/renhyd.19.1.123 }\end{array}$ & $\begin{array}{l}\text { El objetivo de la } \\
\text { investigación fue } \\
\text { determinar la } \\
\text { prevalencia } \\
\text { sobrepeso y obesidad en } \\
\text { estudiantes escolares y } \\
\text { adolescentes del área } \\
\text { Urbana de la ciudad de } \\
\text { Riobambar Los } \\
\text { resultados demostraron } \\
\text { la prevalencia de exceso } \\
\text { de peso } \\
\text { (sobrepeso/obesidad) en } \\
\text { estudiantes escolares y } \\
\text { adolescentes del área } \\
\text { Urbana de la ciudad de } \\
\text { Riobamba es alta } \\
\text { (24,1\%) y es mayor en } \\
\text { escolares (27,7\%) que en } \\
\text { adolescentes (21,5\%). } \\
\text { Son necesarias medidas } \\
\text { de intervención } \\
\text { inmediatas para prevenir }\end{array}$ \\
\hline & & & & $\begin{array}{lcc}\text { y tratar } & \text { este } & \text { grave } \\
\text { problema } & \text { de } & \text { Salud } \\
\text { Pública. } & & \\
\end{array}$ \\
\hline $\begin{array}{l}\text { Borrás, T., Reynaldo, A., } \\
\text { \& López, M., }\end{array}$ & $\begin{array}{l}\text { Adolescentes: } \\
\text { razones para su } \\
\text { atención }\end{array}$ & 2017 & $\begin{array}{l}\text { Correo Científico } \\
\text { Médico, 21(3), 858-875. } \\
\text { Recuperado en 05 de } \\
\text { mayo de 2021, de } \\
\text { http://scielo.sld.cu/scielo. } \\
\text { php?script=sci_arttext\&pi } \\
\text { d=S1560- } \\
\text { 43812017000300020\&ln } \\
\text { g=es\&tlng=es. Indexada } \\
\text { Google académico, Scielo } \\
\text { Latindex }\end{array}$ & $\begin{array}{l}\text { La investigación sobre la } \\
\text { base de la búsqueda } \\
\text { bibliográfica } \\
\text { Pubmed, en } \\
\text { Google Scholar aporta } \\
\text { elementos importantes a } \\
\text { nuestra investigación } \\
\text { Básicamente revisa } \\
\text { estudios publicados, } \\
\text { relacionados con las } \\
\text { razones a tener en cuenta } \\
\text { para brindar una mejor } \\
\text { atención a los } \\
\text { adolescentes, en relación } \\
\text { con aspectos, } \\
\text { conceptuales que } \\
\text { comprometen su futuro } \\
\text { tales como factores de } \\
\text { riesgos, grado de } \\
\text { vulnerabilidad entre } \\
\text { otros. }\end{array}$ \\
\hline Razo, AA., & $\begin{array}{l}\text { Las actividades } \\
\text { recreativas y el } \\
\text { tiempo libre de los }\end{array}$ & (2017) & $\begin{array}{l}\text { Proyecto de Investigación } \\
\text { previo a la Obtención del } \\
\text { Título de Licenciado en }\end{array}$ & $\begin{array}{l}\text { El estudio siguió un } \\
\text { enfoque cualitativo con } \\
\text { el objetivo de demostrar }\end{array}$ \\
\hline
\end{tabular}


adolescentes de 14 a 17 años del caserío San Luis, Cantón Tisaleo"

Sigler, L., Ruano, AO., Palacio, DM, (2020) 9

$\begin{array}{lrl}\text { Programa } & \text { de } & 2020 \\ \text { actividades } & \text { de } & \\ \text { recreación física } & \\ \text { para adolescentes } & \\ \text { del municipio } \\ \text { Encrucijada. Una } \\ \text { necesidad } \\ \text { insoslayable }\end{array}$

Ciencias de la Educación, Mención: Cultura Física https://repositorio.uta.edu .ec Universidad Técnica de Ambato Facultad de Ciencias Humanas y de la Educación. Carrera de Cultura Física Modalidad: Presencial

Universidad Central
"Marta Abreu" de Las
Villas. Facultad de
Cultura Física http:/
/revistaciaf.uclv.edu.cu
Ciencia y Actividad
Física. Volumen 7 No 1
Mes enero. Junio 2020
Pag 48-5
Pag 48-5

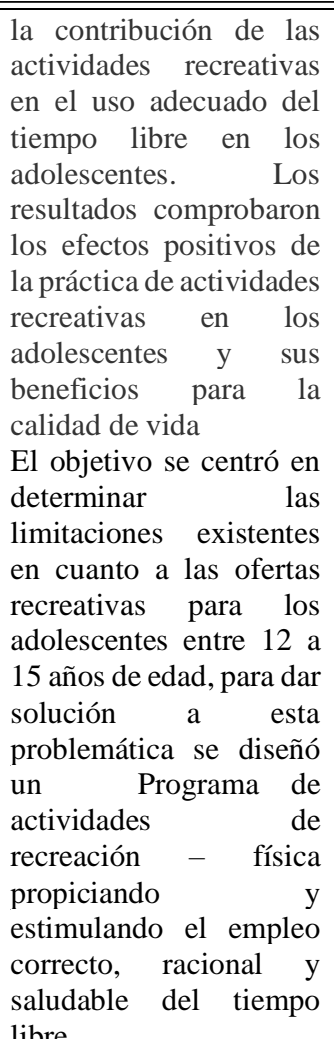

libre.

Derivado de lo anterior se determinó como principales resultados los siguientes:

1. Los estudios y las teorías precedentes confirman que la Recreación y el uso del tiempo libre están muy vinculados con las transformaciones sociales y culturales de las diferentes épocas, es por ello que se requiere que las actividades a desarrollar permitan el desarrollo físico, mental, cultural y social de los participantes. Las mismas han de estar acorde con sus características y contexto sociocultural. Reafirmándose la necesidad de continuar profundizando en el tema objeto de estudio.

2. Se logró profundizar en las generalidades e importancia de la Recreación y su incidencia en la formación integral y en el desarrollo biopsicosocial de los adolescentes, coincidiéndose con los planteamientos de: Pérez, (1997), Lezama, (2000), Waichman (2015), Mateo, (2014), Chávez \& Sandoval (2014), Tocto et al., (2015) Calero et al., (2016) (b), y Razo, (2017), quienes enfatizan en la importancia de la Recreación en las diferentes etapas de la vida, significando su incidencia en el progreso de habilidades físico motoras, sociales, conductuales y cognitivas del adolescente, De igual manera se concuerda con Hernández \& Kelly (2014) y Sosa (2015), al insistir en la necesidad de continuar profundizando en esta línea de investigación específicamente en lo relacionado con las orientaciones a considerar para la implementación en la práctica de las estrategias de intervención mediante la Recreación que satisfagan los intereses y expectativas de los adolescentes.

3. Quedó confirmado la necesidad de trabajar la selección de actividades recreativas desde la perspectiva del diagnóstico en cuanto a gustos, motivaciones e intereses. Chávez \& Sandoval (2014). Resultando esta la vía 
más idónea para el perfeccionamiento de las estrategias de intervención y la implementación en la práctica de proyectos y programas recreativos que permitan tributar de forma efectiva a la formación integral de los adolescentes y por ende a la práctica sistemática de actividades recreativas. Somos de la opinión que la corresponsabilidad que se logre por parte de los entes gubernamentales y educativos, así como de las personas encargadas en desarrollar las estrategias recreativas de intervención, incluyendo los propios adolescentes garantizará el éxito y cumplimiento de los objetivos a lograr.

4. Dentro de las orientaciones a considerar se deben tener en cuenta las siguientes:

- Cumplir con las fases previas a la realización de las actividades. Esto incluye: Diagnóstico, planificación y elaboración, aplicación, valoración y retroalimentación.

- Las actividades a realizar deben estar seleccionadas y planificadas según intereses y gustos de los participantes.

- Deben proporcionar aprendizajes significativos sobre la base de las buenas relaciones interpersonales, el desarrollo biopsicosocial y la formación integral de los adolescentes participantes.

- El personal encargado de la realización de las actividades debe estar capacitado y tener las competencias requeridas para desarrollarlas exitosamente.

\section{Conclusiones}

Se concluye que:

- La adolescencia se caracteriza por ser una etapa de grandes cambios los cuales requieren de atención permanente, no sólo para prevenir la aparición de posibles enfermedades, sino también para potenciar su desarrollo integral.

- La inclusión y participación en actividades recreativas adecuadamente planificadas y en correspondencia con los gustos y preferencias de los adolescentes constituyen una alternativa eficaz para potenciar su desarrollo biopsicosocial.

- La corresponsabilidad que se logre por parte de todas las personas implicadas en desarrollar estrategias recreativas de calidad, así como de los propios adolescentes, garantizará el éxito y cumplimiento de los objetivos a lograr.

\section{Referencias Bibliográficas.}

Aguilar, L., (2004). "La recreación como perfil profesional". Córdoba, Revista. Recreando.

Borrás, T., Reynaldo, A., \& López, M., (2017). Adolescentes: razones para su atención. Correo Científico Médico, 21(3), 858-875. Recuperado en 23 de 
junio de 2021, de http://scielo.sld.cu/scielo.php?script=sci_arttext\&pid=S156043812017000300020\&lng=es\&tlng=es.

Calero, S., Klever, T., Caiza M R., Rodríguez, Á F, \& Analuiza E F.(a), (2016). Influencia de las actividades físico-recreativas en la autoestima del adulto mayor. Revista Cubana de Investigaciones Biomédicas, 35(4), 366-374. Recuperado en 31 de marzo de 2021, de http://scielo.sld.cu/scielo.php?script=sci_arttext\&pid=S086403002016000400007\&lng=es\&tlng=es.

Calero, S., Fernández, A., \& Castillo de la Rosa, FL.,(b), (2016). Recreation activities to improve social behavior. Study in children and adolescents aged 9-14. Revista Cubana de Medicina General Integral, 32(3) Recuperado en 05 de junio de 2021, de http://scielo.sld.cu/scielo.php?script=sci_arttext\&pid=S0864 $21252016000300008 \& \operatorname{lng}=\mathrm{es} \& \operatorname{tlng}=\mathrm{en}$.

Castillo de la Rosa, F. L. (2016). Actividades recreativas para mejorar la conducta social en adolescentes de 12 a 14 años del barrio Guamaní. Maestría en Recreación y Tiempo Libre. Universidad de las Fuerzas Armadas ESPE. Matriz Sangolqui http://repositorio.espe.edu.ec/handle/21000/12651

Chávez, E., \& Sandoval, L., (2014) Estudio del tiempo libre de los adolescentes ecuatorianos del Colegio Nacional Juan de Salinas de Sangolquí. EFDeportes.com, Revista Digital. Buenos Aires, Año18, No 188, Enero de 2014. http://www.efedeportes.com/

Diccionario de la Lengua Española. Edición Tricentenario. Actualización 2020. https://dle.rae.es/

Dos Reis DC, Correa de Almeida TA, Mendes, M., Henrique, R., Faria, AM., Vulnerabilidades a la salud en la adolescencia: condiciones socioeconómicas, redes sociales, drogas y violencia. Rev Latino Am Enfermagem. 2013[citado 26 feb 2016]; 21(2).Disponible en: http://www.scielo.br/pdf/rlae/v21n2/es_01041169-rlae-21-02-0586.pdf

Herrera, S. (2016). Actividad fisica recreativa y calidad de vida en adultos mayores. Fundaciòn UADE. Recuperado el 31 de marzo del 2021, de https://repositorio.uade.edu.ar/xmlui/bitstream/handle/123456789/4079/Herrera. pdf? sequence $=1 \&$ is Allowed $=\mathrm{y}$

Hernández, M., \& K, D., (2014). Eficacia de la aplicación de actividades físicorecreativas, en el desarrollo motriz en los niños de 10-12 años de la Escuela Santa María de los Lojas. http://repositorio.espe.edu.ec/handle/21000/10382 
Ladinez, J., (2020). Actividades físicas - recreativas y la inclusión social con adultos parapléjicos. Tesis en opción al grado de Master en Educación. Mención Inclusión y Atención a la diversidad. Universidad Laica Vicente Rocafuerte. Guayaquil. Obtenido de http://repositorio. http://repositorio.ulvr.edu.ec/handle/44000/4054.

Lezama C. (2000) Papel del recreador en la sociedad contemporánea. Disponible en: http://redcreacion.org/documentos.htm

Mateo, JL. (2014) Las actividades recreativas: sus características, clasificación y beneficios. EFDeportes.com. Revista Digital. Buenos aires, año 19, No 196, Septiembre de 2014. http:// www.efdeportes.com/

Nacional, A. (2015). Ley del deporte, educación física y recreación . Quito. Recuperado el 2 de agosto de 2018

Pérez Sánchez, Aldo. (1997) Recreación: Fundamentos teórico-metodológicos”. México, Inst. Politécnico Nacional para el Instituto Superior de Cultura Física "Manuel Fajardo", Departamento de Recreación y Turismo.

Ramos A E., Ojeda, R., Báez, D., Nuñez, E., (2011) La Recreación: necesidad y oportunidad para el desarrollo humano desde y para las comunidades. EFDeportes.com, Revista Digital. Buenos Aires, Año 16, No 160, Septiembre de 2011. http:// www.efedeportes.com

Ramos, P., Carpio, A., Delgado, V., Villavicencio, V., (2015). Sobrepeso y obesidad en escolares y adolescentes del área urbana de la ciudad de Riobamba, Ecuador. Revista Española de Nutrición Humana y Dietética, 19(1), 2127. https://dx.doi.org/10.14306/renhyd.19.1.123

Razo, AA., (2017), Las actividades recreativas y el tiempo libre de los adolescentes de 14 a 17 años del caserío San Luis, Cantón Tisaleo" Universidad Técnica de Ambato Facultad de Ciencias Humanas y de la Educación. Carrera de Cultura Física Modalidad: Presencial Proyecto de Investigación previo a la Obtención del Título de Licenciado en Ciencias de la Educación, Mención: Cultura Física https://repositorio.uta.edu.ec

Tocto JG, Herrera VH, Altamirano M, Valle JG, Maqueira GC. Motivación de la comunidad estudiantil universitaria hacia la práctica de actividades físicodeportivas. Estudio en Chimborazo. Lecturas: Educación Física y Deportes. 2015 Noviembre; 20 (210):1-12.

Torres, DE., Castillo AF., Fonseca, Ingrid., (2016). Beneficios de la recreación dirigida en la clase de educación física de los estudiantes del grado décimo del colegio Celestin Freinet URI: http://hdl.handle.net/10656/4475

Organización Mundial de la Salud. (2021) Informe presentando en conmemoración al Día Mundial de la Obesidad. https://www.iusc.es/noticias/370-dia- 
mundial-de-la-obesidad-2021.

Sigler, L., Ruano, AO., Palacio, DM, (2020) Programa de actividades de recreación física para adolescentes del municipio Encrucijada. Una necesidad insoslayable Universidad Central "Marta Abreu" de Las Villas. Facultad de Cultura Física http:/ /revistaciaf.uclv.edu.cu Ciencia y Actividad Física. Volumen 7 No 1 Mes enero. Junio 2020 Pag 48-59

Sosa, D. A. (2015). Gestión de los procesos físico-recreativos a partir del CETiLReF, la Disciplina Recreación Física y el Departamento de Recreación Física del INDER de Villa Clara. "II Taller Nacional, Actividad Física y Desarrollo Local”. Conferencia Magistral. S. Spíritus (.8-13)-04-2015

UNICEF. Obesidad - Infantil (2021) https://www.unicef.org/ecuador/obesidadinfantil.

Waichman, PA., (2015) Recreación: Educación o Pasatismo? De la alienación a la Libertad. Quadernsanimacio.net N ${ }^{\circ} 21$; Enero de 2015 
PARA CITAR EL ARTÍCULO INDEXADO.

Cristhian Steve, M. H., Steven Mauricio, C. N., \& María Lorena, S. J. (2021). La Recreación en los adolescentes. Su importancia en el desarrollo biopsicosocial: Una aproximación teórica -descriptiva. Explorador Digital, 5(3), 110-125. https://doi.org/10.33262/exploradordigital.v5i3.1787

\section{LCiencia}

El artículo que se publica es de exclusiva responsabilidad de los autores y no necesariamente reflejan el pensamiento de la Revista Explorador Digital.

El artículo queda en propiedad de la revista y, por tanto, su publicación parcial y/o total en otro medio tiene que ser autorizado por el director de la Revista Explorador Digital.
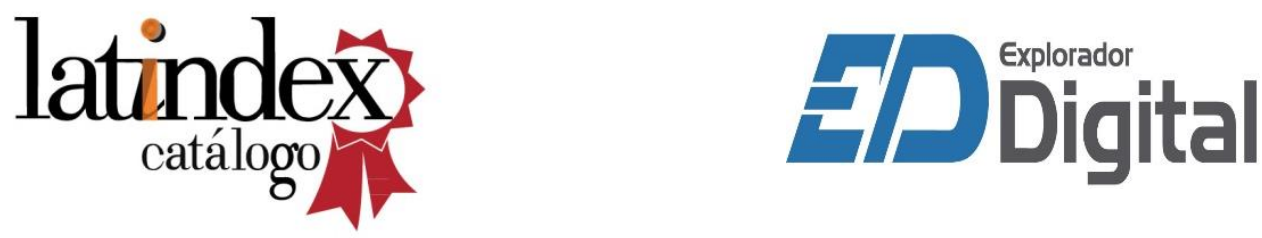